\begin{tabular}{|c|c|c|c|}
\hline 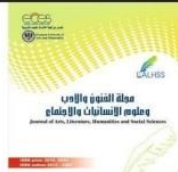 & 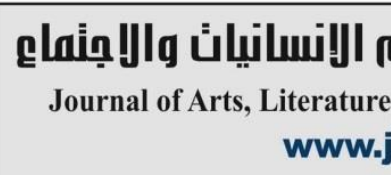 & $\begin{array}{l}\text { es and Social Sciences } \\
\text { m }\end{array}$ & \\
\hline$=\quad=$ & Volume (70) August 2021 & العدد (70) أغسطس 2021 & \\
\hline
\end{tabular}

\title{
Impact of Social Media on Communication Skills as Expressed by Gau Students
}

\author{
Academic Researcher IBRAHIM HOROUB \\ Girne American University- Communication Faculty - PHD, Communication and \\ Media Management- North Cyprus \\ Email: avihroub1951994@gmail.com
}

\begin{abstract}
This quantitative study aims to identify the impact of social media platforms on communication skills. It is necessary to investigate the negative and positive effects of virtual reality as expressed by youths, as well as identifying the thoughts, attitudes, and beliefs the youths adopt when using this kind of virtual interaction. Therefore, quantitative research methodology was adopted to determine if there is a significant difference in spending many hours on social media platforms based on communication skills among GAU students by using SPSS. In order to test the hypotheses of this current study, three types of statistical test were used to get accurate results in this regard. Consequently, One-way ANOVA, Two-way ANOVA, and Independent- Samples $\mathrm{T}$ Test were applied to investigate the hypotheses. Accordingly, the sample of this study consisted of 150 students at GAU. However, due to special circumstances, it was better to adopt online questionnaire as a method of data collection than face to face encounters. The statistical analysis reveals that social media platforms negatively influence the ability of young people to communicate and use body language effectively in real life situations. Moreover, there is a strong evidence for a significant interaction of using body language skills and academic performance of youths based on spending too much time on social media platforms. However, there is no significant difference between males and females in spending many hours on social media platforms. Therefore, gender cannot be considered a crucial factor in this context. The study also indicates that human behavior cannot be controlled by a single factor, but rather by multiple factors. Whatever the causes of behavior change, social media platforms have a role to play, depending on the variables of the environment, content, means, audience and interaction.
\end{abstract}

Keywords: Communication skills, Social media platforms, Human behavior, SPSS, Technology, Virtual reality, New media. 


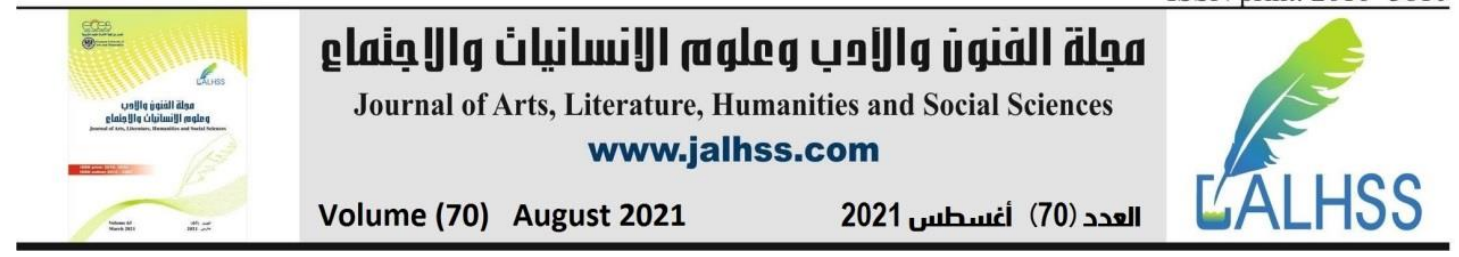

\section{CHAPTER 1}

\section{INTRODUCTION}

Social media platforms are important tools for improving social structures, sharing ideas and visions among individuals, and understanding the cultures of different people, in addition to their active and distinctive role as successful means of communication at different levels. The development of communications goes hand in hand with technology, which increases the importance of social networks and creates the need to build appropriate strategies to promote these communication tools (Tosun, 2012).

It is an integrative process in which individuals discuss different topics openly to exchange information and create common meanings and interpretations in order to reach consensus and influence each other's behavior and attitude. The process of interaction in social networks is not affected by the barriers of time and space. Social networking therefore creates a kind of social dynamics (Tosun, 2012). Consequently, social networking has become a global phenomenon due to its great impact on people of different ages, cultures and different levels of education. Bartlett (2006 ) emphasizes that social networking sites are a set of applications that increase people's interaction in sharing and expressing common ideas, and creating virtual groups to exchange information and social data.

We all know that the development of technology innovations in recent years has been accompanied by a noticeable development in communication systems, and consequently, this has a marked impact on communication channels in terms of their characteristics, the content they provide, and the way the receiver interacts with the message. So, in order to make communication between individuals or institutions more effective, the appropriate channel should be carefully chosen to carry the appropriate message. In this context, it can be said that social media platforms are the most rich medium because users can send messages, whether written or audio, and share photos, videos and other forms of information more easily than other communication channels do (Lengel and Daft, 1988).

Accordingly, we understand ourselves through mass media by exploring other realities, watching people who have similar social and cultural conditions, watching how they face different situations, and thus we can identify ourselves, the roles we should play, the characters we want to imitate, and the new lifestyles and experiences that we would like to learn in the future (De fleur and Ball rokeach, 1989).

Mass communication outlets usually provide us with a flow of information about different issues, so they influence our understanding, our attitudes, and our various judgments. Mass media affect the cognitive system of individuals through the longterm exposure as a source of information, distorting the existing background of values and beliefs, and building new perceptions and cognitive backgrounds (Bandura, 1994). 
Sociology and psychology have been significantly interested in studying the impact of mass media -including social media platforms- on human beings. These means can control the intellectual process of each person by teaching him a new pattern of thinking or changing his perceptions and attitudes about life and society. Therefore, the media as part of the daily lives of individuals contribute to influencing them regardless of their age, gender, and psychological conditions. The role of these media is to build the social beliefs for individuals and identify their roles, attitudes, and interests (Bates, 1975).

\subsection{Statement of the Problem}

There is a debate among scholars regarding the impact of social media networks on the cultural patterns and communication skills. Joinson (2008) said that social media have a direct negative impact on society where they weaken the real social communication and interaction between individuals and affect the social values and norms. On the other hand, social networking is a virtual social structure that develops the communication skills of its users in which people who share the same interests want to exchange knowledge or specific material through audio, video, or text via electronic means (Bartlett, 2006 ).

Mass media are one of the most important tools that contribute to manufacturing consciousness of individuals in a particular environment and making them able to acquire a set of information and knowledge about the society they live in (Chomsky and Herman, 1988). Therefore, these means and tools have its great influence on the communication skills in a clear and direct manner as well as they can affect the behavioral patterns of people in different circumstances, especially with the recent developments in both traditional and social media.

It can be assumed that when adopting a new innovation such as social media, this innovation should be significantly compatible with the user's values, culture and lifestyle so that this model becomes attractive to large segment of people. At this point it is important to state that users of social media platforms are not aware of the real effect or effects of these communication tools on their communication skills in real life encounters. This fundamentally raise a debate about the impact of this new technology and the ability of individuals to make a balance between using these means on the one hand and controlling their behavior and attitudes on the other hand.

\subsection{Purpose of the Study}

This research aims to clarify the negative and positive effects of social media platforms on communication skills as expressed by youths, as well as identifying the thoughts and beliefs the youths adopt when using this kind of virtual interaction. By discussing the concept of new media, it would also be better to examine the role of educational institutions as well as family in directing the individual's behavior and encouraging him to interact with the social environment rather than the virtual world of social media. 


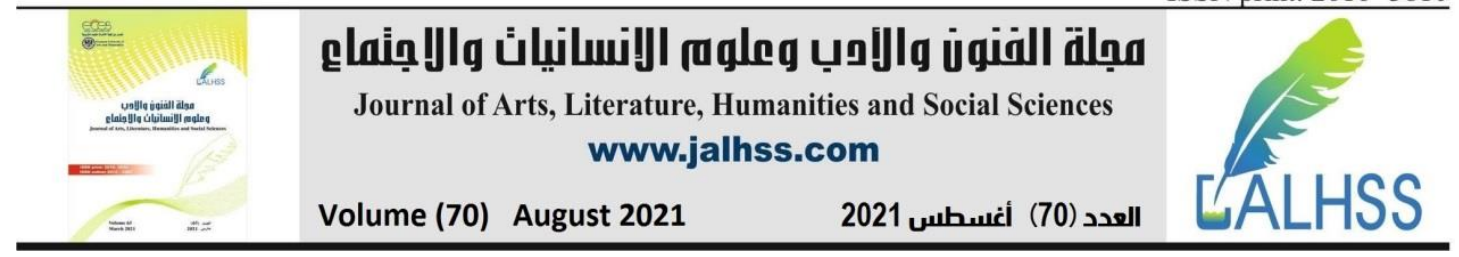

\subsection{Significance of the Study}

The Internet has become an important communication medium in this century and an integral part of our daily lives. Its use is not limited to a particular age group, but it is largely used by both young and adult. Because the Internet is an essential part of social and family lives, young guys grow up in the Internet environment, especially with immediate access to social media and the availability of the network in small electronic devices all the time.

Therefore, this current research has been conducted to determine the benefits of using social media platforms by youths and the harmful effects as well. Consequently, this increases the importance of study, especially with regard to the new concepts, assumptions, and theories related to social media platforms.

\subsection{Research Questions}

a. What is the impact of new technology and social media on communication skills?

b. How to avoid the negative effects of social media on youths and enhance their social interaction. Which techniques should be used to achieve this purpose?

c. Why do young people spend long hours on social networking platforms, while they can avoid virtual reality by actually communicating face-to-face?

d. What is the role of new communication systems in the evolution of human behavior in postmodern societies?

e. To what extent social media platforms can be regulated at the internal and external levels and how to avoid its negative impact among youths such as addiction?

\subsection{Hypothesis}

H1. There is a statistically significant difference in spending many hours on social media platforms based on using body language skills in face to face conversations.

H2. There is a significant interaction of using body language skills and academic performance of youths on spending too much time on social media platforms.

H3. There is a statistically significant difference in spending many hours on social media platforms based on declining activates with family and friends among youths.

H4. There is a statistically significant difference between males and females in spending many hours on social media platforms.

\subsection{Assumptions}

a. Social networking platforms affect behavior patterns and communication skills negatively, especially among young people.

b. The new media have a greater impact on individuals than traditional media. 


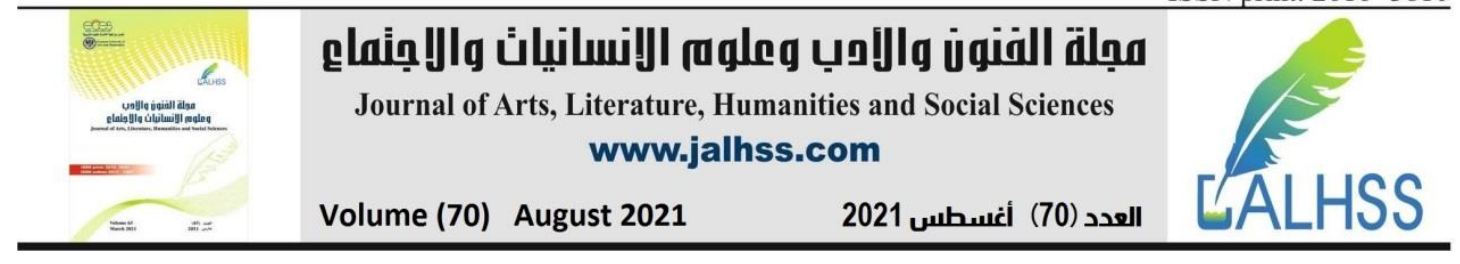

c. Over time social networking platforms contribute to changing the social behavior of young people and this may create a form of addiction among them.

d. Social networking platforms negatively affect the academic performance of young people, especially if they spend too much time on using them every day.

\subsection{Limitations}

This quantitative study was basically conducted to understand the impact of social media platforms on communication skills among youths. However, there was not enough time to test a large sample to get accurate results. Therefore, the researcher selected a representative sample of the undergraduates at GAU. In addition, there were other factors and contexts that affected the research procedures, for example the diversity of cultures and ethnicities among GAU students, which play a key role in determining the appropriate method and approach to examine their communication skills at the psychological and social levels.

With regard to theoretical frameworks that address the phenomenon of social media, this study adopted two theories that are closely related to the main subject. These theories are diffusion of innovations theory and social network analysis theory. These two theoretical frameworks can be applied to understand the effect of social media on young people, whether negative or positive. The first theory focuses on the mechanism that users adopt a new innovation like Social Media and the factors and contexts that significantly impact the popularity of this innovation. Furthermore, the second theory highlights the nature of relationships and interactions among social network users. However, it was necessary to use a third theory to explain the effect of social media on users in terms of their behavior and communication skills that they adopt throughout face to face encounters. Consequently, because there is no specific theory that investigates the influence of social media, the powerful media effects theory was adopted to examine the assumptions of this current study, considering that social media platforms are a form of mass communication. 


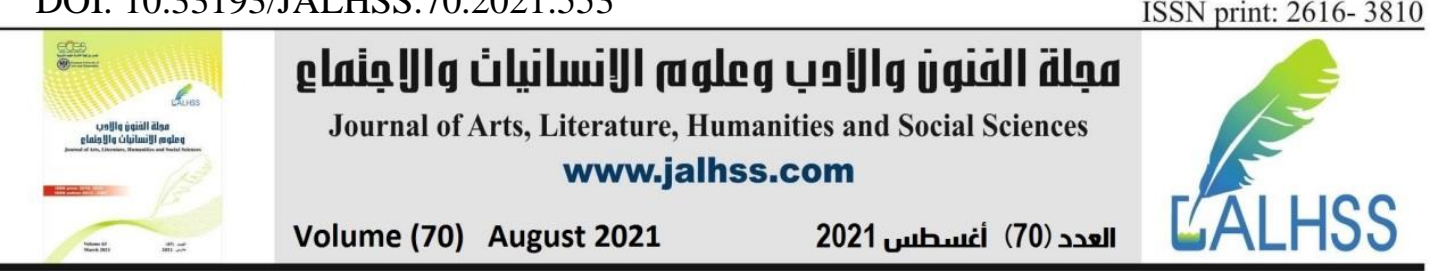

\section{CHAPTER 2}

\section{LITERATURE REVIEW AND THEORETICAL FRAMEWORK}

\subsection{Literature Review}

Mass media in the present age play a large role in societies. They represent political, economic, and social patterns in a way that can directly affect individuals. The new communication systems have the ability to remarkably effect and influence the audience based on assumptions related to psychology, sociology, and other sciences.

Mass communication systems play an influential role in controlling society in which individuals receive information, opinions and attitudes from the media that aim to shape selective attentions, interpretations, and perceptions. The media affect the cognitive process because they provide information in a way that can convince the target audience of certain facts. In other words, the media are one of the most important tools that contribute to manufacturing consciousness of individuals in a particular environment and making them able to acquire information about the society they live in (Jakubowicz \& Plamer, 2002). Also, Garbner et.al., (1979) discussed that mass media are the source of most images and widely exchanged messages. The symbolic world presented by mass media is an important source of knowledge and contributes to the development of meanings and behaviors among individuals. The contribution of media to change values is homogenous within the various social groups. The media also change these perceptions of social reality and modify them with other forms and patterns.

Mecheel's study (2010) aimed to identify the impact of the use of social networks on social relations. The study was applied to a sample of 1,600 young users of social access networks in Britain. The results showed that more than half of the adults who use sites including Facebook and YouTube have admitted that they spend more time on the Internet than they spend with their true friends or family members. The study also showed that they talk less on the phone, do not watch television much, play fewer computer games, and send a large amount of text messages as well as mailing. The study also found that about $53 \%$ of sample demonstrated that online social networks have already changed their lifestyles. Half of Internet users in the UK are members of a social networking site compared to $27 \%$ Only in France, $33 \%$ in Japan and $40 \%$ in the US.

Kujath (2011) also conducted a study which aimed to reveal whether social networking is complementary to, or a substitute for, real-world relations. The study sample consisted of 183 undergraduate students in Washington, DC, and all of them had a Facebook account. To measure the degree to which this platform contributed to maintaining existing personal relationships, specific questions were asked about the number of friends they did not meet personally. The results indicated that individuals tend to use the social network to stay in touch with people they already know. Oh, et.al., (2014) also selected a sample of 339 participants to investigate the relationship between supportive interactions on social networking sites and people's perception of 


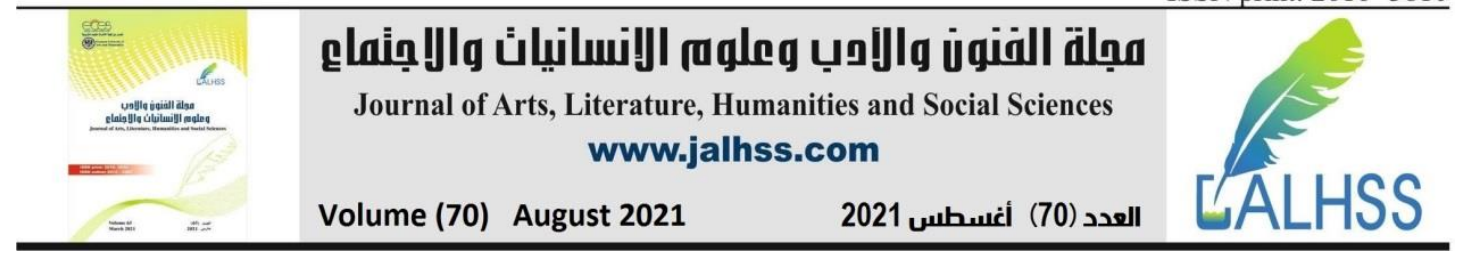

social support, feelings of belonging, and satisfaction with life. The study also investigated the relationship between social support and direct impact of social media on individuals over 5 days. The analysis revealed a positive relationship between supportive interaction and positive impact after interaction.

In addition, there is an important study aimed to reveal the relationship between the use of the Facebook platform and self-esteem and satisfaction as well as social and emotional compatibility. The study used 70 study students from Boston universities. The researchers used questionnaires that included measures of the nature of communication on Facebook for the number of friends and intensity of use, as well as measures of self-esteem and social and interpersonal compatibility. The results of the study indicated that there is a negative relationship between the number of friends on Facebook and the social and academic compatibility of the first year students, and that the excessive time spent on the platform is associated with low self-esteem. Also, the results of the study indicated that the number of friends online in the master's students is positively associated with social harmony, and this is due to the improvement of social relations among these students after spending years in the university (Kalpidou et.al., 2011)

Moreover, a study by Valenzuela, et.al. (2009) aimed to investigate the relationship between the use of Facebook by students and attitudes and behaviors that improve their social relations. The study sample consisted of 2603 university students in Texas in random selection method. The researchers developed a measure of the use of Facebook. They also used four criteria to check satisfaction with life, social confidence, and civil and political participation, and applied these measures to the study sample by using the survey analysis method. The study found a positive correlation between the use of Facebook and the satisfaction level of life, social confidence, civic participation and political participation. The researchers recommended ignoring concerns about the negative effects of Facebook.

On the other side, Karbinsiki (2010) conducted a study in New York to identify the impact of using Facebook on the academic level of students. The researcher adopted the social survey method and the questionnaire as a tool for collecting information. The study found that the grades obtained by students who are addicted to use Facebook, the largest social network on the Internet, are much lower than those obtained by their classmates who do not use this website. The results also showed that the more time a student spends on the site, the lower his grades in the exams, since the addiction to Facebook negatively affect their academic achievement.

\subsection{Theoretical framework}

With regard to theoretical frameworks that address the phenomenon of social media, this study adopted three theories that are closely related to the main subject. These theories are diffusion of innovations theory, social network analysis theory, and strong media effects theory. Diffusion of innovations is one of the most important 


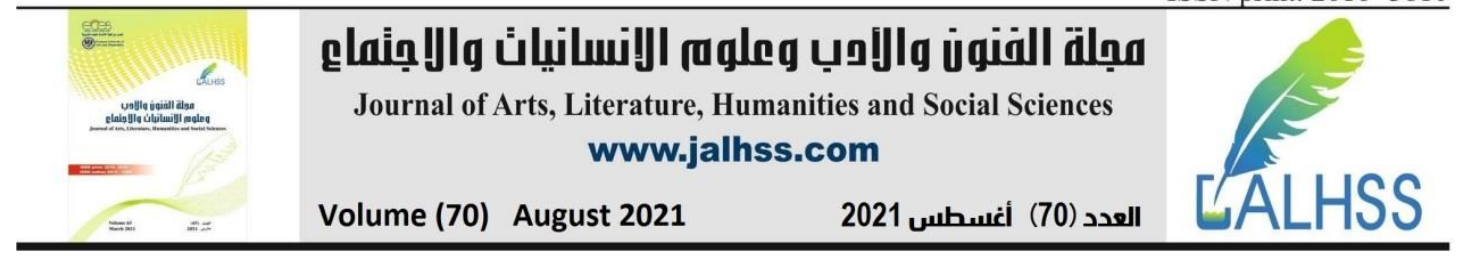

theories in communication studies to assess the effectiveness and spread of inventions in a specific social framework. These innovations include new technological devices, ideas, channels, applications, social platforms, and communication systems. Therefore, this theory has an important role in revealing the extent to which users adopt a new innovation and the extent of its competition among other alternatives in different social contexts and systems. The theory of diffusion of innovations depends on a number of assumptions that explain and articulate the process of adopting the new innovation by users and the development of its popularity over time.

Rogers (2003) who developed the diffusion of innovations theory in the early 1960s argues that most of new innovations and technologies have been adopted based on certain standards or criteria that determine the popularity and efficiency of these news ideas or practices among individuals. Therefore, the first characteristic that should be taken into consideration is the ability of innovation to achieve the special goals and needs of adopters. Each innovation is distinguished from other competitors in terms of speed, efficiency, performance, cost, quality and ease. So, it can be said that social media platforms are more popular than traditional media outlets because these new platforms significantly allow users either individuals or institutions to easily interact, share, communicate, and generate content.

The communication system of social media is based on a complex relationships and interactions between numbers of users. This assumption is important when social network analysis takes place. Therefore, it can be said that relationships via social media platforms can be divided into a set of nodes that represent the content and information exchanged between the users. This connection and continuous interaction contributes to revealing the patterns of communication and the different roles played by each component of this electronic network. There are different roles that users play through the network. For example, a person or organization may be a link or a bridge between other people and other institutions that do not have connections before. The second important role that some users play on networks is the gatekeeping. This functional role is effective when it is intended to control and monitor the flow of information between users as a form of power at the internal and external levels. The more the network contains a large number of members, the more complicated the process of analyzing communication patterns and reactions, and vice versa (Monge and Contractor, 2001).

The authors of the powerful influence theory suggest that mass media have strong and direct influence on the public. Mass media have the ability to change attitudes, opinions, and values of individuals. The authors of this theory build their perceptions based on psychological and social assumptions. Regarding the psychological aspect, it was discussed that people are often driven by emotions and instincts that mass communication can easily control them. If the mass communication can inject its audience with certain information that simulate these instincts, they will be directly affected by this injection. People rely on the media as a key system to understand the social environment around them. In other words, media are a major source on which 


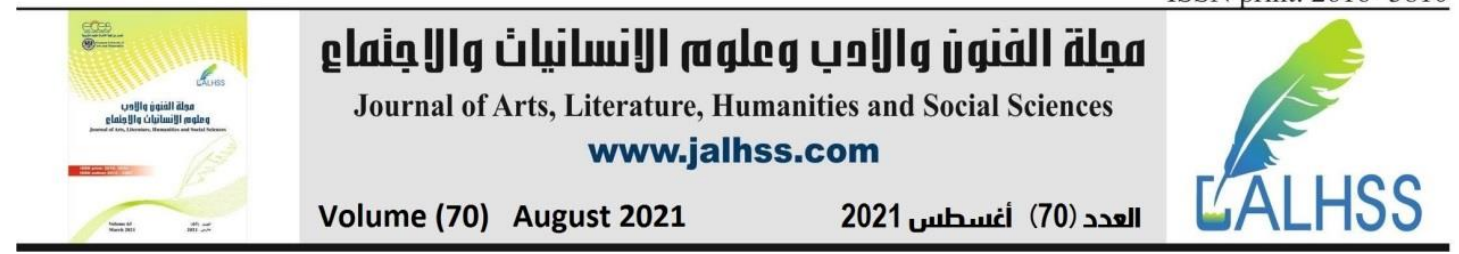

the public relies to get information and understand what is around them at the social and cultural levels (Noelle, 1979).

\section{CHAPTER 3 \\ METHOD}

This chapter contains a description of the research design and research philosophy alongside information on the sampling and data collection techniques used.

\subsection{Research Design}

This current study was conducted to investigate the negative and positive effects of social media on youths. Quantitative research methodology was adopted to determine if there is a significant difference in spending many hours on social media platforms based on communication skills among GAU students. In many cases, quantitative studies are better than qualitative studies when it comes to test hypotheses that address the phenomenon related to new media, human behavior, and effective communication skills.

\subsection{Sampling Method and Data Collection}

The researcher selected a representative sample of the undergraduates students at GAU. In this regard the sample consisted of 150 students at faculty of communication. However, due to the special circumstances related to corona virus outbreak, it was better to adopt online questionnaire as a method of data collection than face to face encounters. The following table shows the distribution of males and females from the total sample:

gender

\begin{tabular}{|ll|r|r|r|r|}
\hline & \multicolumn{1}{|c|}{ Frequency } & Percent & Valid Percent & Cumulative Percent \\
\hline Valid & male & 72 & 48.0 & 48.0 & 48.0 \\
& female & 78 & 52.0 & 52.0 & 100.0 \\
& Total & 150 & 100.0 & 100.0 & \\
\hline
\end{tabular}

\subsection{Method of Data Analysis}

Online questionnaire was conducted to answer the questions of this study. Accordingly, SPSS was used to analyze the impact of social media platforms on youths. In order to test the hypotheses of this current study, three types of statistical test were adopted to get accurate results in this regard. Consequently, One-way ANOVA was used to test hypotheses 1 and 3 whereas Two-way ANOVA was used to test hypothesis 2. Finally, Independent- Samples T Test was used to investigate hypothesis 4 . 


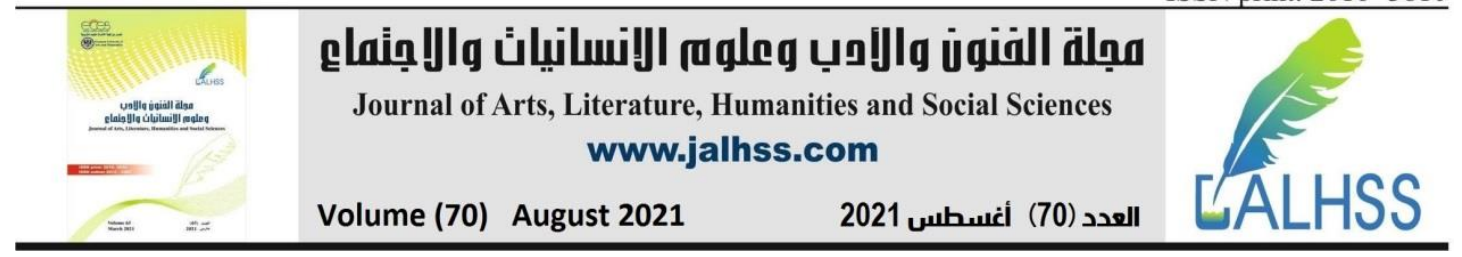

\section{CHAPTER 4}

\section{RESULTS AND DISCUSSION}

The results of this quantitative study highlight the impact of social media on communication skills and how they affect the youth's activates with friends and family as well as demonstrating specific skills by using body language in face to face conversations and real life situations.

Hypothesis 1. There is a statistically significant difference in spending many hours on social media platforms based on using body language skills in face to face conversations.

In this regard, One-way ANOVA was used to test hypotheses 1. By using SPSS the results show that $\mathbf{P}$ value $\mathbf{( 0 . 0 0 0 0 1 )}<$ alpha $(\mathbf{0 . 0 5})$, so we reject the null hypothesis and find strong evidence for a statistically significant difference in spending many hours on social media platforms based on using body language skills in face to face conversations. In this case, the answer is absolutely yes. Social media platforms influence the ability of young people to communicate effectively and use body language in real life situations. The following tables show the results of hypothesis 1 by SPSS.

Test of Homogeneity of Variances

Spending many hours on social media

\begin{tabular}{|c|c|c|c|}
\hline Levene Statistic & df1 & $\mathrm{df} 2$ & Sig. \\
\hline 16.551 & 3 & 146 & .0002 \\
\hline
\end{tabular}

ANOVA

Spending many hours on social media

\begin{tabular}{|l|r|r|r|r|r|}
\hline & Sum of Squares & \multicolumn{1}{|c|}{ df } & Mean Square & F & Sig. \\
\hline Between Groups & 159.057 & 3 & 53.019 & 9.438 & $\mathbf{0 0 0 0 1}$ \\
Within Groups & 820.203 & 146 & 5.618 & & \\
Total & 979.260 & 149 & & & \\
\hline
\end{tabular}

Hypothesis 2. There is a significant interaction of using body language skills and academic performance of youths on spending too much time on social media platforms.

Two-way ANOVA was used to test hypothesis 2. By using SPSS the results show that $\mathbf{P}$ value $(\mathbf{0 . 0 0 1})$ < alpha $(\mathbf{0 . 0 5})$, so we reject the null hypothesis and find strong evidence for a statistically significant interaction of using body language skills and academic performance of youths on spending too much time on social media platforms. Most of GAU students who spend too much time on social media platforms 


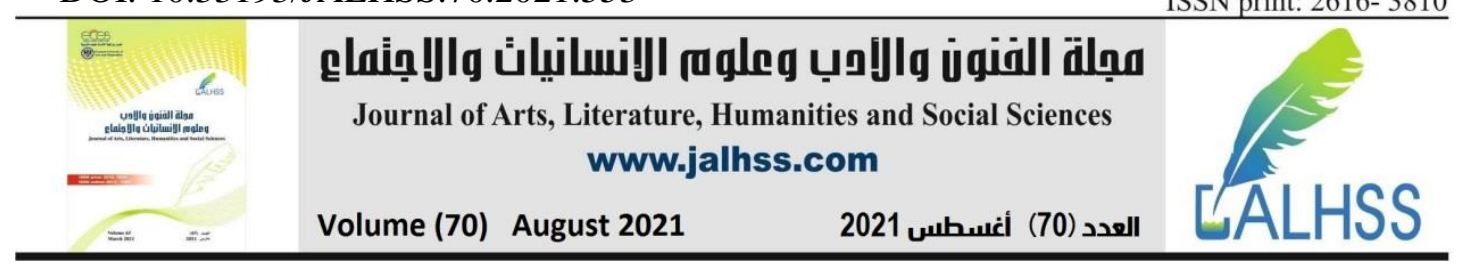

use less body language skills when communicating with other people and this in turn impacts their academic performance. In this case, the answer is yes, the result is statistically significant. The following tables show the results of hypothesis 2 by SPSS.

Tests of Between-Subjects Effects

Dependent Variable: Spending many hours on social media

\begin{tabular}{|l|r|r|r|r|r|}
\hline Source & $\begin{array}{c}\text { Type III Sum of } \\
\text { Squares }\end{array}$ & df & Mean Square & \multicolumn{1}{c|}{ F } & Sig. \\
\hline Corrected Model & $353.260^{\mathrm{a}}$ & 11 & 32.115 & 7.080 & .000 \\
Intercept & 2228.852 & 1 & 2228.852 & 491.344 & .000 \\
Academic performance & 95.663 & 3 & 31.888 & 7.030 & .000 \\
body language & 148.137 & 3 & 49.379 & 10.885 & .000 \\
Academic performance * & 105.544 & 5 & 21.109 & 4.653 & $\mathbf{. 0 0 1}$ \\
body language & 626.000 & 138 & 4.536 & & \\
Error & 5451.000 & 150 & & & \\
Total & 979.260 & 149 & & & \\
Corrected Total & & & & \\
\hline
\end{tabular}

a. $\mathrm{R}$ Squared $=.361$ (Adjusted R Squared $=.310$ )

Between-Subjects Factors

\begin{tabular}{|ll|c|c|}
\hline & & Value Label & $\mathrm{N}$ \\
\hline Academic performance & 1.00 & often & 33 \\
& 2.00 & sometimes & 60 \\
body language & 3.00 & seldom & 21 \\
& 4.00 & never & 36 \\
& 1.00 & often & 60 \\
& 2.00 & sometimes & 30 \\
& 3.00 & seldom & 27 \\
\hline
\end{tabular}

Hypothesis 3. There is a statistically significant difference in spending many hours on social media platforms based on declining activates with family and friends among youths.

Statistically, One-way ANOVA was used to test hypotheses 3. By using SPSS the results show that $\mathbf{P}$ value $(\mathbf{0 . 0 0 7})<$ alpha $(\mathbf{0 . 0 5})$, so we reject the null hypothesis and find strong evidence for a statistically significant difference in spending many hours on social media platforms based on declining activates with family and friends among youths. In this case, the answer is absolutely yes, the result is statistically significant. GAU undergraduates students can physically abandon contact with their families and friends because they use social networking sites. The following tables show the results of hypothesis 3 by SPSS.

Test of Homogeneity of Variances

Spending many hours on social media

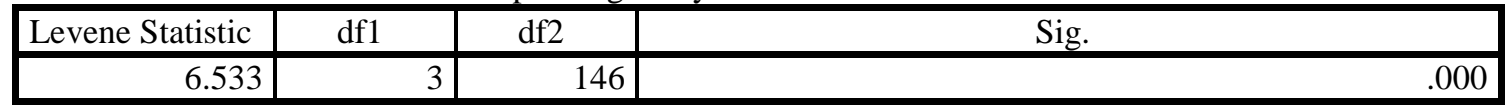




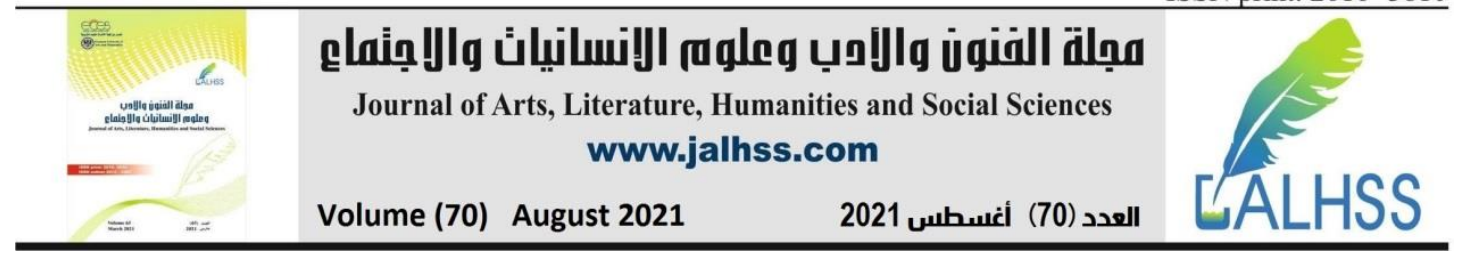

ANOVA

Spending many hours on social media

\begin{tabular}{|l|r|r|r|r|r|}
\hline & Sum of Squares & \multicolumn{1}{|c|}{ df } & Mean Square & F & Sig. \\
\hline Between Groups & 77.171 & 3 & 25.724 & 4.163 & \\
Within Groups & 902.089 & 146 & 6.179 & & \\
Total & 979.260 & 149 & & & \\
\hline
\end{tabular}

Hypothesis 4. There is a statistically significant difference between males and females in spending many hours on social media platforms.

Statistically, Independent- Samples T Test was used to investigate hypothesis 4 . By using SPSS the results show that $P$ value (0. 084) > alpha (0.05), so we fail to reject the null hypothesis and find that there is not enough evidence for a significant difference between males and females in spending many hours on social media platforms. In other words, gender cannot be considered a crucial factor in this context, because the behavioral patterns of both males and females are often similar. Male and female spend equal time when using social media platforms, whether to chat with friends or family or to meet new people and share with them certain opinions and experiences. The following table shows the results of hypothesis 4 by SPSS.

Group Statistics

\begin{tabular}{|ll|r|r|r|c|}
\hline & gender & N & \multicolumn{1}{|c|}{ Mean } & Std. Deviation & \multicolumn{1}{c|}{ Sig. } \\
\hline Spending many hours on & male & 72 & 5.0833 & 2.77184 & 0.084 \\
social media & female & 78 & 5.8077 & 2.31908 & \\
\hline
\end{tabular}

Other descriptive results related to the current questionnaire. Which of the social networking sites are most popular for GAU students?

The results show that Instagram $(48 \%)$ is the most popular social networking site among GAU students, then Facebook (30\%), What's up (14\%), and Twitter (8\%) respectively. The following table shows the percentages by SPSS.

Which one is your favorite?

\begin{tabular}{|ll|r|r|r|r|}
\hline & Frequency & Percent & Valid Percent & Cumulative Percent \\
\hline Valid & Facebook & 45 & 30.0 & 30.0 & 30.0 \\
& 12 & 8.0 & 8.0 & 38.0 \\
& twitter & 72 & 48.0 & 48.0 & 86.0 \\
Instagram & 21 & 14.0 & 14.0 & 100.0 \\
What's up & 150 & 100.0 & 100.0 & \\
Total & & & \\
\hline
\end{tabular}

\section{What motivates you to use/log into your social network account?}

The results show that chatting with friends (38\%) is the first motivation among GAU students to use social media platforms, then kill time (22\%), play games (22\%), and post photos $(18 \%)$ respectively. The following table shows the percentages by SPSS. 


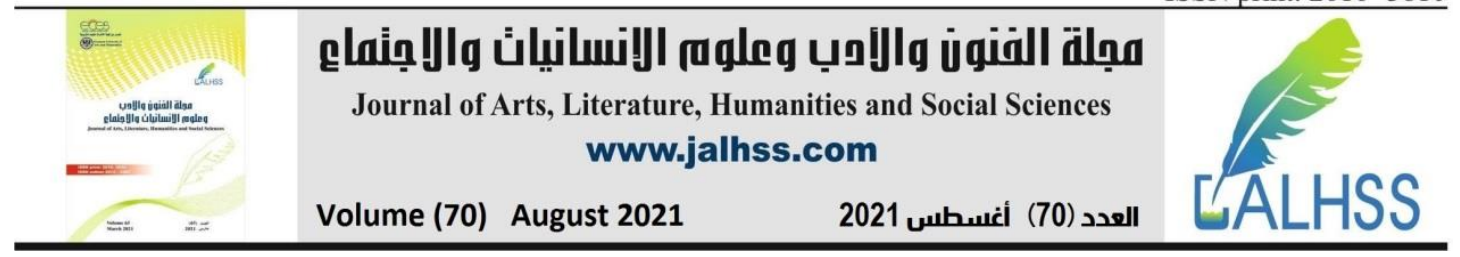

Motivation

\begin{tabular}{|ll|r|r|r|r|}
\hline & Frequency & Percent & Valid Percent & Cumulative Percent \\
\hline Valid & post photos & 27 & 18.0 & 18.0 & 18.0 \\
& kill time & 33 & 22.0 & 22.0 & 40.0 \\
play games & 33 & 22.0 & 22.0 & 62.0 \\
chatting & 57 & 38.0 & 38.0 & 100.0 \\
Total & 150 & 100.0 & 100.0 & \\
\hline
\end{tabular}

\section{CHAPTER 5}

\section{CONCLUSIONS AND RECOMMENDATIONS}

\subsection{Conclusions}

The results show that social media platforms such as Facebook and Instagram make it easier for youths to communicate with their friends and relatives and learn about social norms and values. However, social networking sites have negative effects on face to face communication skills. Consequently, the most common negative impact of social networking sites is addiction. This kind of addiction fundamentally affects other important activities among youths such as reading, social interaction, waking up early, exercise, and so on. Social media platforms also have the ability to affect the prevailing values and norms that an individual adopts and have at the same time a greater role in destroying them and replacing them with other forms and patterns. The study also indicates that social media platforms influence the ability of young people to communicate effectively and use body language in real life situations. There is a strong evidence for a statistically significant interaction of using body language skills and academic performance of youths on spending too much time on social media platforms. Most of young people, who spend too much time on social media platforms, use less body language skills when communicating with other people and this in turn may impact their academic performance. Furthermore, some students can physically abandon contact with their families and friends because they heavily use social networking sites. There is strong evidence for a significant difference in spending many hours on social media platforms based on declining activates with family and friends among youths. However, there is no significant difference between males and females in spending many hours on social media platforms. Therefore, gender cannot be considered a crucial factor in this context because the behavioral patterns of both males and females are often similar.

\subsection{Recommendations}

Additional studies are significantly needed to investigate the impact of social media platforms on communication skills especially the use of body language by youths in face to face conversations. It is also better to examine the content presented by social 


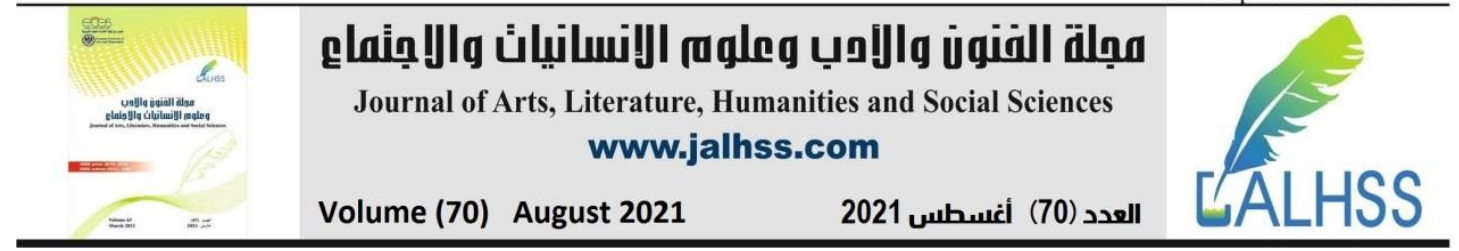

media platforms and determine whether young guys are affected by these new social, political, and cultural patterns promoted my social media.

\section{REFERENCES}

1. Bandura, Albert. (1994). Social Cognitive Theory of Mass Communication. Erlbaum.

2. Bartlett-Bragg, A. (2006). Reflections on pedagogy: Reframing practice to foster informal learning with social software, Training and Development in Australia, 32, (5), 21-24.

3. Bates, Thomas R. (1975). Gramsci and the Theory of Hegemony. Journal of the History of Ideas. P. 351-366.

4. Chomsky, Noam, \& Edward S. Herman. (1988). Manufacturing consent: the political economy of the mass media. New York: pantheon Books.

5. De fleur, M. and Ball rokeach, S.(1989). Theories of mass communication. New York: Longman.

6. Gerbner, G., Gross, L., Morgan, M., Signorielli, N., \& Jackson-Beeck, M. (1979). The Demonstration of Power: Violence Profile. No. 10. Journal of Communication, 29, P.177-196.

7. Jakubowicz, A., and Plamer, R. (2002). Framing Suharto: Australian media accounts of the fall from power. International Communication Gazette, 64 (2), 199 213.

8. Joinson, A. N. (2008). Looking at, 'looking up' or 'keeping up with' people? Motives and uses of Facebook. In CHI 2008 Proceedings. New York: ACM Press.

9. Kalpidou M, Costin D, Morris J. (2011). The relationship between Facebook and the well-being of undergraduate college students. Cyberpsychol Behav Soc Netw, 14(4):183-9.

10. Karbinsiki, Aren. (2010). Facebook and the technology revolution. New York: Spectrum Publications.

11. Kujath. C. (2011). Facebook and Myspace: complement or substitute for face-toface interaction? Cyber psychology, Behavior, and Social Networking, 14(12): 75-78.

12. Lengel, R.H., \& Draft, R.L. (1988). The Selection of Communication Media as an Executive Skill. Academy of Management Executive, 2(3), 225-232.

13. Mecheel, V. (2010). Facebook and the invasion of technolog(ical communities. NY: New York.

14. Monge, P.R. \& Contractor, N.S. (2001). Emergence of communication networks. In F. Jablin \& L. L. Putnam (Eds.), The new handbook of organizational communication (pp. 440-502). Thousand Oaks, CA: Sage.

15. Noelle, Neuman. (1979). Return to the concept of powerful mass media. Quoted in communication theories: origins. Methods and uses. By W. Severin and J.W. Tankard (N.Y. House), P.257-58.

16. Oh, H., Ozkaya,E .\& LaRose, R. (2014). How does online social networking enhance life satisfaction? The relationships among online supportive interaction, 


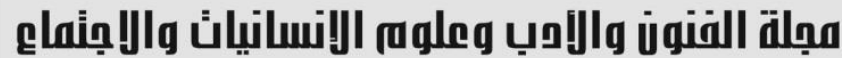 \\ Journal of Arts, Literature, Humanities and Social Sciences www.jalhss.com

affect, perceived social support, sense of community, and life satisfaction. Computers in Human Behavior, (30). 69-78.

17. Rogers, E.M. (2003). Diffusion o innovations ( $5^{\text {th }}$ ed.). New York: Free Press.

18. Tosun, L. (2012). Motives for Facebook use and expressing "true self" on the internet. Computers in Human Behavior, (28), 1510-1517.

19. Valenzuela, S., Park, N. \& Kee, K. (2009). Is there social capital in a social network site?: Facebook use and college students' life satisfaction, trust, and participation. Computer-Mediated Communication, 14 (4) : 875-901. 